Revue de l'Institut des langues et cultures

d'Europe, Amérique, Afrique, Asie et Australie

14 | 2011

Traduction et Ergonomie

\title{
Poste du travail du traducteur et responsabilité : une question de perspective
}

The Translator's Work Station and Responsibility: a Question of Perspective

\section{Daniel Toudic et Guillaume de Brébisson}

\section{OpenEdition}

\section{Journals}

Édition électronique

URL : http://journals.openedition.org/ilcea/1043

DOI : $10.4000 /$ ilcea.1043

ISSN : 2101-0609

\section{Éditeur}

UGA Éditions/Université Grenoble Alpes

\section{Édition imprimée}

ISBN : 978-2-84310-203-5

ISSN : 1639-6073

Référence électronique

Daniel Toudic et Guillaume de Brébisson, «Poste du travail du traducteur et responsabilité : une question de perspective », ILCEA [En ligne], 14 | 2011, mis en ligne le 30 juin 2011, consulté le 01 mai 2019. URL : http://journals.openedition.org/ilcea/1043; DOI : 10.4000/ilcea.1043

Ce document a été généré automatiquement le 1 mai 2019.

(c) ILCEA 


\title{
Poste du travail du traducteur et responsabilité : une question de perspective
}

\author{
The Translator's Work Station and Responsibility: a Question of Perspective
}

Daniel Toudic et Guillaume de Brébisson

1 Depuis l'article fondateur de Martin Kay «The Proper Place of Men and Machines in Language Translation" (Kay, 1980, 1997), le "poste de travail informatique du traducteur » a souvent été décrit et étudié. Dans cet article, Kay établissait les principes de ce qui pour lui devait être la relation idéale entre le traducteur et la machine. Kay y décrit ce qu'il appelle "the translator's amanuensis» ou le "serviteur du traducteur ", appellation qui traduit clairement la perception « humaniste » de Kay, pourtant l'un des fondateurs de la linguistique computationnelle :

The kind of translation device I am proposing will always be under the tight control of a human translator. It is there to help increase his productivity and not to supplant him. It will never resort to ad hoc measures that have not been explicitly sanctioned by him. In its normal and recommended mode of use, it will appeal to him rather than being forced back on unfounded guesses. (Kay, 1980, p. 19)

2 Plus de vingt ans plus tard, Daniel Gouadec (2003, 2009, p. 363-364) nous projetait dans un avenir de science fiction, qui l'est de moins en moins à mesure que la vision qu'il ébauche se fait plus précise : celle du traducteur devenu simple valideur de solutions défilant sur un écran virtuel qui lui permet, croit-il, d'accéder par la seule pensée à toutes les ressources cognitives, terminologiques et phraséologiques produites depuis le début de l'humanité (alors que, parfaitement inutile, il est devenu à son insu simple objet d'étude pour des scientifiques curieux de découvrir les réactions de leur cobaye).

3 Entre ces deux visions, à peine trente ans d'évolution du poste de travail du traducteur ont profondément transformé la relation du traducteur avec son outil de travail, ses donneurs d'ordre, les matériaux traduits et sa façon de traduire. 


\section{Ergonomie : une question de perspective}

4 La vision que décrit Daniel Gouadec résume à elle seule tout le paradoxe de l'évolution du poste de travail du traducteur. Celle-ci est en effet perçue et vécue très différemment, selon que l'on se place dans la perspective du traducteur, de l'entreprise ou du service de traduction, ou encore de l'utilisateur final de traductions.

\section{Perspective du traducteur}

5 Pour le traducteur, qu'il soit salarié ou indépendant, un poste de travail parfaitement ergonomique est un outil qui lui permet de disposer immédiatement, sans changer d'environnement, avec la même interface, de tous les éléments qui vont lui permettre de se consacrer à ce qu'il juge être son cœur de métier : le transfert linguistique. Autrement dit, d'obtenir dans l'instant où les questions se posent, toutes les réponses d'ordre cognitif, documentaire, terminologiques ou purement linguistiques qui vont permettre de dérouler sans heurts et sans interruption une traduction répondant à tous les critères d'exactitude informationnelle, de validité terminologique et de fluidité de lecture. L'ergonomie idéale permet d'appeler à tout instant tous les éléments internes et externes au document source permettant d'éclairer la nature de tel concept, les connotations de tel figement, le sens de tel enchaînement logique, y compris par la visualisation. Elle permet de disposer à tout instant des concordants terminologiques possibles en contexte, de façon à effectuer les choix les plus justes, et d'accéder à une base de documents authentiques faisant autorité dans le domaine, fournissant les ressources phraséologiques indispensables. Elle permet aussi de récupérer instantanément les traductions existantes de segments ou de paragraphes récurrents stéréotypés afin d'accélérer le travail et d'assurer l'harmonisation interne du document cible. Mais avant tout, ce poste de travail idéal laisse la main au traducteur, qui en devient le pilote et non l'exécutant.

\section{Perspective de l'entreprise ou du service de traduction}

Pour une organisation fournissant des prestations de traduction, qu'elle soit commerciale ou de service public, l'ergonomie idéale du poste de travail du traducteur est celle qui permet de réduire le coût de la pré- et de la post-traduction tout en accélérant la phase de transfert.

7 Dans l'entreprise, le poste de travail doit permettre de recevoir tous les formats de fichiers, d'en faire une analyse rapide, d'identifier ce qui est déjà traduit ou n'est pas à traduire, et de dispatcher les lots à traduire vers des traducteurs en interne ou en externe en sachant qu'ils disposent de la même configuration informatique et des mêmes outils, de pouvoir leur mettre à disposition rapidement et facilement les ressources terminologiques et documentaires dont ils vont avoir besoin et de réduire au maximum le temps de relecture, d'harmonisation et/ou de révision nécessaire. La capacité à disposer de tous les outils adéquats est encore plus indispensable dans les secteurs, tels la localisation (des logiciels, des jeux vidéos, des applications mobiles, etc.) qui exigent des applications dédiées. Plus généralement, le traducteur doit être à même d'assurer un maximum de prestations (harmonisation terminologique et phraséologique, vérification 
linguistique, voire pré-formatage) : autant de tâches que l'entreprise n'aura pas à prendre en charge.

\section{Perspective du donneur d'ouvrage / utilisateur final}

8 Le donneur d'ouvrage (client commercial, service client) ou l'utilisateur final, quant à lui, se préoccupe beaucoup plus de l'ergonomie des matériaux traduits que de celle du poste de travail permettant de les produire. Il souhaite surtout disposer de ces matériaux dans les délais fixés, au rapport qualité/prix le plus avantageux et dans un format qui permette leur utilisation immédiate et leur intégration sur les supports appropriés. Toute évolution qui favorise le respect de ces critères sans augmenter le prix des prestations lui est bien sûr agréable. Il n'envisage bien évidemment pas de modifier sa propre configuration informatique ou ses modes de production documentaire pour satisfaire aux contraintes techniques du prestataire.

\section{L'évolution du poste de travail : son impact sur les acteurs}

9 En fonction des critères définis plus haut, les évolutions technologiques de ces vingt dernières années ont été perçues différemment par chacun des acteurs.

\section{Traducteurs indépendants}

Les rapports entre les traducteurs indépendants et les outils technologiques ont essentiellement traversé deux phases. La première (la «lune de miel »), qui s'étend du milieu des années 1980 à la fin des années 1990, est vécue avant tout comme une avancée globalement positive et libératrice. Rappelons qu'il y a vingt-cinq ans à peine, la notion d'ergonomie se limitait pour la majorité des traducteurs à la forme de leur fauteuil et de leur table de travail, sur laquelle trônait, dans le meilleur des cas, une machine à écrire électrique «à boule » permettant de fabuleux gains de productivité. Une étude sur la traduction automatique (TA) constatait encore en 1992 que : «nombre de traducteurs travaillent encore avec le dictaphone et le papier (fiches terminologiques, dictionnaires, versions papier de la traduction après saisie par une secrétaire...) »(Roudaud, 1992, p. 12).

11 Même équipé d'un traitement de texte rudimentaire, le traducteur doit alors constamment passer d'un environnement à un autre, se déplaçant physiquement pour recevoir ou transmettre ses commandes, pour trouver les données terminologiques ou documentaires nécessaires, stockant ses traductions et ses données terminologiques sur différents supports, papier ou numériques, corrigeant manuellement les erreurs ou incohérences terminologiques au prix d'un labeur fastidieux et parfois aléatoire.

À partir des années 1990 jusqu'au début des années 2000, la généralisation de postes de travail informatiques plus performants avec une réelle capacité de stockage des données est donc vécue par la grande majorité des traducteurs comme une avancée majeure dans l'ergonomie de leur environnement et de leurs pratiques de travail. Les avancées technologiques (messagerie électronique, Internet grand public, moteurs de recherche ultra-rapides, bases terminologiques ou de connaissances numériques...) donnent un accès rapide aux données et rendent possible l'envoi des traductions à partir d'un même 
lieu physique et d'un même environnement informatique. Les applications bureautiques de plus en plus sophistiquées permettent d'effectuer en un clic toutes les opérations de vérification linguistique ou de mise en forme et de gérer facilement commandes, projets et factures. Le choix de ces outils reste pour l'essentiel à la discrétion du traducteur, si ce n'est qu'il est, comme tous les utilisateurs, soumis aux évolutions des systèmes d'exploitation et des «standards » commerciaux. Le choix d'un fournisseur d'accès, d'un navigateur et des outils de recherche ne lui est pas imposé. Ses choix restent dictés par les seuls critères de coût et d'ergonomie, d'où la montée en puissance rapide de Google auprès des traducteurs, dès 1998, séduits par la rapidité et la puissance de l'outil et le caractère dépouillé de son interface utilisateur.

Dans les entreprises de traduction, les traducteurs salariés travaillent dans un environnement intégré, avec les mêmes outils standards ou personnalisés par l'entreprise, accédant à des bases terminologiques et à des mémoires de traduction «maison ». En même temps, dans les grands services linguistiques, le poste de travail du traducteur est de plus en plus intégré dans des systèmes complexes, dont l'un des exemples les plus aboutis est le système Euramis de la Direction générale de la traduction à Bruxelles, en cours d'élaboration depuis 1995.

La deuxième phase commence dès les années 2000, avec la généralisation des outils de traduction assistée par ordinateur (TAO) et notamment des systèmes de gestion de mémoires de traduction. Tant que ceux-ci restent confidentiels, ou réservés aux « grands comptes", ils semblent offrir la promesse de gains de productivité substantiels et l'élimination des harmonisations a posteriori fastidieuses et coûteuses en temps. Leur arrivée massive dans les agences à partir des années 2000-2003, avec l'offensive des leaders du marché, marque une rupture. Désormais le traducteur passe progressivement du statut de prescripteur et d'acteur à celui d'opérateur travaillant dans un cadre et avec des outils et des méthodes qu'il n'a pas choisis. Ses méthodes de travail elles-mêmes changent fondamentalement: on lui demande désormais de traduire dans le cadre contraint des phrases pré-segmentées de la mémoire de traduction, avec une interface qui n'a souvent rien de convivial et dont il ne maitrise pas l'ergonomie. L'ergonomie de sa traduction elle-même s'en ressent, puisqu'il ne peut plus, la plupart du temps, donner libre cours à sa capacité de reformulation et de réorganisation au sein d'un même paragraphe, mais doit suivre le découpage effectué par le donneur d'ordre, qui souhaite pouvoir vérifier rapidement l'exactitude et l'exhaustivité de la version traduite.

Pour bon nombre de traducteurs chevronnés, ces années sont vécues comme une période de perte progressive d'autonomie et de responsabilité. D'où la réticence de certains à adopter spontanément les outils de plus en plus nombreux et sophistiqués disponibles sur le marché (ou disponibles gratuitement à travers les logiciels libres ou collaboratifs). Le manque d'engouement technologique s'explique bien sûr également par des considérations plus terre à terre. Les études menées aussi bien en Grande-Bretagne (Fulford et Grenell-Zafra, 2004) qu'en France (SFT, Gouadec), ou à un niveau européen (Höcker et Wheatley, 2003) montrent que les traducteurs indépendants recherchent un retour immédiat sur l'investissement financier ou l'investissement en temps que les technologies nécessitent. Les outils qui demandent un apprentissage plus poussé (outils dédiés de gestion de terminologie, certains outils de PAO) ou dont le coût est jugé prohibitif (certains systèmes de gestion de mémoires de traduction) ne sont utilisés régulièrement que par une minorité de traducteurs. L'enquête eCoLoRe (Höcker et Wheatley, 2003, p. 1) révèle que seuls $29 \%$ des traducteurs britanniques et allemands 
consultés utilisent les systèmes de mémoires de traduction quotidiennement, et $36 \%$, jamais. Pour ce qui concerne l'utilisation des outils de TAO en général, $38 \%$ utilisent des outils de recherche ou de gestion de terminologie, $14 \%$ des outils de PAO et $7 \%$ seulement des logiciels spécifiques de localisation.

Au cours des cinq dernières années, ces chiffres ont régulièrement augmenté. Mais si les traducteurs indépendants se tournent aujourd'hui de plus en plus fréquemment vers ces outils plus sophistiqués ou plus coûteux, c'est parce que le marché, et les clients, l'exigent. L'enquête annuelle de la SFT en France (SFT, 2010, p.30) démontre une progression régulière de l'utilisation de la TAO chez les indépendants français (progression de 65 à $69 \%$ d'utilisateurs entre 2008 et 2009). L'enquête révèle deux tendances en apparence contradictoires : d'une part, l'utilisation croissante de l'outil SDLTrados (67 \% d'utilisateurs parmi les répondants, selon l'enquête de la SFT, voire 90 \% si l'on inclut les produits SDLX), et, d'autre part, une dispersion accrue des outils disponibles et utilisés, qu'il s'agisse de pionniers comme Wordfast ${ }^{1}$, de logiciels " libres " comme Wordfast Anywhere ou OmegaT, de nouveaux venus tels que MemoQ ou d'outils "propriétaires» tels que Translation Workspace de Lionbridge. La contradiction n'est qu'apparente, car si les traducteurs indépendants travaillant avec les agences se voient le plus souvent imposer l'emploi de SDL Trados, ils peuvent aussi rechercher des solutions moins coûteuses ou répondant mieux à leurs préférences lorsqu'ils travaillent avec des clients directs. L'interopérabilité croissante des systèmes de gestion de mémoire permet d'ailleurs de concilier plus facilement les exigences de l'agence avec les préférences du traducteur.

Wordfast s'est d'ailleurs, depuis sa conception, placé dans la perspective du traducteur jaloux de son indépendance et soucieux de préserver son « libre arbitre » professionnel :

Une fois l'ensemble des fonctionnalités de la version Classic transféré à la version Pro, le produit redeviendra une option de choix pour le traducteur indépendant qui souhaite ne pas être inféodé à la suite SDL Trados et disposer d'un outil indépendant des plateformes hébergées de plus en plus communes dans le monde de la traduction (Lionbridge, SDL Trados, Google Translation Toolkit, Across, etc.).

(Anonyme, 2009) ${ }^{2}$

18 Face à ces velléités d'indépendance, les grands concepteurs et distributeurs des «standards» du marché ont rapidement compris qu'ils devaient emboîter le pas aux nouveaux venus s'ils voulaient développer leur marché au-delà des entreprises qui constituent leur clientèle traditionnelle, en s'adressant au public beaucoup plus vaste des indépendants. SDL Trados met désormais l'accent, dans la promotion des produits freelance, sur l'ergonomie et les fonctionnalités destinées à faciliter la vie du traducteur :

Environnement de traduction convivial et simple d'emploi affichant aussi peu de balises que possible, qui vous permet de vous consacrer entièrement à la traduction de vos documents et d'accroître votre productivité.

Tous les formatages, balises, marquages et variables à votre portée. Grâce à des suggestions intelligentes adaptées à votre contenu source, vous pouvez désormais traduire très facilement tout type de fichier. (SDL Trados, 2011)

\section{Prestataires de services linguistiques}

19 La perception des évolutions technologiques par les entreprises et prestataires de services linguistiques est initialement similaire à celle des traducteurs, mais diverge rapidement lorsque les intérêts des premiers commencent à prendre le pas sur ceux de leurs traducteurs sous-traitants. Les entreprises et organisations ont, comme les 
traducteurs indépendants, largement bénéficié de la révolution numérique des années 1990, qui leur a donné accès à de nouveaux marchés nationaux et internationaux et leur a permis de bénéficier des avancées dans les applications bureautiques et les outils de gestion terminologiques et de gestion de projets et de clientèle. Les entreprises les plus puissantes ou les grands services linguistiques mettent en place pendant cette période des ressources terminologiques et phraséologiques de plus en plus nombreuses et exhaustives et les premiers outils d'accès en ligne. Certains investissent déjà depuis le milieu, voire le début des années 1990, dans les premiers systèmes de gestion des mémoires de traduction et dans l'intégration croissante des outils sur le poste de travail. La généralisation de ces outils à partir de 2000, coïncide, pour ces prestataires, avec le début d'une phase d'expansion rapide. Celle-ci s'appuie, justement, sur les nouvelles marges de manœuvre (et marges bénéficiaires potentielles) que leurs confèrent ces outils. La possibilité d'analyser rapidement les documents sources et de déterminer avec précision les volumes à traduire et les taux de répétition leur permet de mieux contrôler la productivité de leurs traducteurs salariés et de diminuer le coût de la sous-traitance. L'alignement et la segmentation permettent d'accélérer le pointage, la relecture et la révision des textes traduits, allant dans le sens de l'allègement recherché des frais de postédition.

21 D'une position de demandeur tributaire de la bonne volonté du traducteur, l'entreprise de services linguistiques devient désormais prescriptrice, ne travaillant qu'avec les traducteurs équipés du système de gestion de mémoires de traduction standard acheté et imposé par l'entreprise ou pouvant travailler avec les systèmes « maison » imposés par certains grands comptes. L'ergonomie du poste de travail du traducteur devient l'affaire du donneur d'ordre autant que celle du traducteur, dans la mesure où elle a une incidence directe sur la productivité des traducteurs salariés et/ou des sous-traitants et sur celle de l'entreprise: tout temps passé par l'entreprise à convertir des fichiers dans un autre format, à extraire du texte ou des éléments non textuels, puis à réinjecter des segments traduits dans un environnement donné, est du temps perdu et de la marge bénéficiaire en moins.

Les rapports avec les traducteurs externes changent donc radicalement : il ne s'agit plus uniquement de confier le travail à celle ou celui qui traduit le mieux, mais à celles et ceux parmi ces bons traducteurs, qui disposent des outils adéquats et savent s'en servir de la façon la plus efficace. La véritable entreprise de traduction cesse d'être seulement l'intermédiaire entre un client et un prestataire, mais devient un prestataire à part entière imposant ses conditions et ses critères de qualité et de productivité aux traducteurs sollicités.

Plus récemment, les derniers développements en matière de plateformes "SaaS " ("

Software as a Service »), tout en semblant donner un regain d'autonomie aux traducteurs en matière d'outils de travail, du fait de l'interopérabilité accrue des systèmes de mémoires de traduction et de la gestion de formats de fichiers multiples, renforcent de fait le contrôle du donneur d'ordre sur l'ensemble des collaborateurs engagés sur un projet donné. Ainsi, l'accès en temps réel aux ressources terminologiques partagées, s'il permet de substantiels gains de productivité, peut enlever au traducteur la plus-value que constitue son expertise dans un domaine donné ou sa compétence à rechercher et à exploiter les sources pertinentes, en redonnant la primauté aux experts "non linguistes", comme le laisse entendre la description suivante du système " Translation Workspace » de la société Lionbridge : 
Centralized, live terminology assets allow virtual teams of content authors, linguists and subject matter experts and to synchronize terminology creation and updates in real time. Glossaries can be created and updated in real-time, ensuring that every member of the team always has the latest terminology at their fingertips. (Lionbridge, 2011) de la configuration du poste de travail du traducteur ni du processus qui mène à la production de la traduction, la situation commence à changer avec l'apparition de demandes spécifiques d'utilisation d'interfaces ou d'outils propriétaires imposés par le client. L'exemple suivant illustre certaines des nouvelles contraintes et problématiques auxquels peuvent donner lieu ces situations.

Le contrôle sur les sous-traitants est de fait accru par les systèmes d'accès par abonnement, qui permettent de gérer en temps réel les temps d'accès de chaque utilisateur :

Sophisticated license management functionality allows subscribers to float access licenses between users as needed. This allows subscribers to scale the number of concurrent users accessing their subscription at any time, without having idle users consuming valuable access. (Lionbridge, 2011)

Si le traducteur a effectivement accès en temps réel à des quantités inégalées de ressources documentaires et terminologiques, la qualité de sa production et sa productivité sont contrôlées à chaque instant par le donneur d'ordre grâce aux fonctionnalités sophistiquées de gestion et de suivi que permet le système :

Translation Workspace can track TM contributions on a per user basis. It provides a mechanism to track productivity by supplier, by language, by assets, or any combination thereof. (Lionbridge, 2011)

\section{Les donneurs d'ouvrage et utilisateurs finaux}

Un client institutionnel a développé à grands frais un site d'information grand public et une interface de saisie des données. Souhaitant faire traduire le site dans plusieurs langues, il confie le travail à un prestataire extérieur, en imposant pour la phase de traduction l'utilisation de la même interface de saisie à utiliser directement en ligne. Mais si l'ergonomie de cette dernière est bien adaptée à l'entrée des données dans la langue d'accueil du site, elle s'avère particulièrement peu conviviale et mal adaptée lorsqu'il s'agit de traduire ces mêmes données. Parmi les défauts relevés : l'impossibilité de trier les articles à traduire par domaines ou par sujets, l'impossibilité de connaitre le statut d'un texte donné pendant le processus de traduction (en cours de traduction, de révision, de contrôle ou validé ?), la fragmentation et la dispersion des textes à traduire, la disparition du texte à traduire au fur et à mesure de sa traduction, rendant impossible tout retour en arrière ou vérification a posteriori, et la manipulation malaisée des liens et des balises. Très rapidement, les sous-traitants mobilisés se rendent compte qu'ils passent beaucoup plus de temps sur cette traduction qu'avec les outils et méthodes habituels. Certains exigent de ce fait une renégociation des tarifs initialement fixés, ou une modification des processus employés. Plusieurs traducteurs sous-traitants préfèrent même interrompre leur contrat plutôt que de poursuivre le travail dans des conditions qu'ils jugent inacceptables. Au final, le prestataire est obligé de transiger en supprimant l'étape de la révision croisée, entraînant de ce fait un risque accru d'incohérence terminologique et phraséologique; un pourcentage plus élevé de textes est par conséquent soumis au contrôle qualité, avec une augmentation proportionnelle du coût 
pour le prestataire et le client (d'environ $40 \%$ ) et la productivité stagne à environ 1500 mots par jour, soit moitié moins du rendement attendu d'un traducteur professionnel. Au vu de ces difficultés et des surcoûts entraînés par l'inadaptation de l'outil, le client accepte de revoir l'interface de traduction, en prenant en compte les demandes du prestataire et de ses sous-traitants, et la traduction peut enfin se poursuivre dans de meilleures conditions.

Cet exemple illustre bien la difficulté de faire accepter par les traducteurs, comme par n'importe quel utilisateur, un outil dont l'ergonomie impose un apprentissage et une prise en main progressifs et longs, peu compatibles avec la réalisation d'un projet soumis à des contraintes de délais et de productivité. Il illustre surtout les dangers d'une déconnexion entre les informaticiens concepteurs d'outils et d'interfaces et les traducteurs qui les utilisent. Ainsi, une ergonomie mal maîtrisée ou inadaptée aux contraintes particulières d'un projet de traduction donné entraîne retards et augmentation des coûts, là où une concertation préalable aurait permis de les éviter.

\section{Un risque de déresponsabilisation et de désengagement}

Les évolutions que nous venons de décrire vont par conséquent toutes dans le même sens : celle d'une perte d'autonomie de responsabilité du traducteur vis-à-vis de son outil de travail et, partant de là, vis-à-vis de sa traduction.

Le prescripteur technologique est désormais le donneur d'ordre ou le donneur d'ouvrage. C'est lui qui impose le système et les mémoires à utiliser, restreignant du même coup considérablement les marges de manœuvre du traducteur à la fois dans ses propres choix technologiques et dans sa pratique traductive. Au fil des années, cette contrainte s'est alourdie. D'abord limitée au choix du système, le traducteur utilisant les mémoires de traduction constituées par lui-même, ou par un seul traducteur, elle s'est rapidement étendue à l'utilisation de mémoires partagées, notamment avec les systèmes dédiés imposés par les grands acteurs du marché. Non seulement le texte source est parfois réduit à des segments parcellaires, isolés de leur contexte, mais le traducteur peut se trouver confronté, soit à une traduction existante insatisfaisante qu'il ne peut pas modifier (ou qu'il n'a aucun intérêt à modifier car elle ne lui sera pas payée), soit à des variantes de traduction multiples proposées par des prestataires différents, sans connaître les critères qui lui permettraient de choisir la plus pertinente.

31 Parfois, c'est le format du document source fourni par le client direct ou indirect qui va poser des problèmes au traducteur dans son utilisation du système de gestion de mémoires de traduction. Un document Word comportant de nombreux schémas légendés ou des tableaux complexes peut devenir un cauchemar pour le traducteur, obligé de traquer le texte source invisible, ou dont le segment cible disparait en arrière plan. Le problème de la conversion des PDF reste encore un problème récurrent, même s'il est désormais en voie de résolution dans les nouvelles versions des systèmes de gestion de mémoires de traduction.

Dans d'autres cas, comme nous l'avons vu plus haut, c'est l'ensemble de l'interface utilisateur fournie par le client qui s'avère complètement inopérant et contre productif. Ces cas illustrent parfaitement le paradoxe actuel: à force de vouloir réduire le traducteur à un simple exécutant, et à le déresponsabiliser par rapport au produit final 
qu'est la traduction, le donneur d'ordre peut dans certains cas réduire la productivité du traducteur et la qualité de sa production au lieu de l'améliorer. Si la recherche d'une meilleure ergonomie du poste de travail a pour seul but de réduire le traducteur à un exécutant robotisé, elle est souvent contre-productive pour tous les acteurs concernés.

La généralisation en cours de la traduction automatique risque d'aggraver encore plus rapidement cette tendance. Toute la plus-value technique du système étant accaparée par les concepteurs/distributeurs et, dans une moindre mesure, par les experts chargés de paramétrer et d'optimiser le système pour chaque projet de traduction, le rôle du "traducteur " peut rapidement se réduire à celui de postéditeur chargé de valider et, dans la vision la plus optimiste, de réviser les segments automatiquement traduits.

\section{Conclusion}

Le traducteur deviendra-t-il de fait un operative, pour reprendre le nom donné aux ouvriers du textile au xix siècle et dans la première moitié $d u x^{e}$ siècle, subissant la même évolution que celle qui marqua le passage de la filature et du tissage artisanal à l'ère industrielle? Verra-t-on, comme dans cette industrie, une hiérarchisation s'établir entre, en bas de l'échelle, les « petites mains » chargées de réparer les imperfections de la traduction signalées par le système, comme les enfants chargés de réparer les fils cassés dans les ateliers de filature, et en haut « l'aristocratie ouvrière " chargée de paramétrer les machines ou bien de réviser ou récrire «à la main » les traductions jugées encore trop complexes ou trop sensibles pour être confiées entièrement à la machine. Parallèlement aux industries de la langue de plus en plus concentrées et puissantes, existera-t-il encore un artisanat d'art réservé aux traducteurs recherchés pour leur compétences ou leurs spécialités particulières, comme il existe des maîtres reconnus dans les métiers artisanaux?

L'avenir du "traducteur " passe donc sans doute par une réévaluation radicale de son rôle dans le processus global de la traduction. Le traducteur humain devra être considéré, comme Kay l'avait pressenti, ou comme Gouadec le préconise (Gouadec, 2005), comme un acteur de l'ensemble du processus, y compris en aval et en amont du seul transfert. La qualité d'une traduction "assistée » (avec mémoires de traduction) dépend en grande partie de la possibilité pour le traducteur de pouvoir modifier certains segments déjà traduits en fonction des éléments de connaissance nouveaux (ou de nouvelles ressources terminologiques ou phraséologiques) inconnus ou non disponibles au moment de la première traduction de ces segments. La qualité d'une traduction automatisée, dépendra in fine de la capacité du traducteur à intervenir à chaque phase du processus : validation $\mathrm{du}$ dictionnaire terminologique et phraséologique (avec sélection des champs d'application et ajout du dictionnaire particulier de la traduction en cours), paramétrage des spécifications linguistiques et du dictionnaire de normalisation, validation des segments traduits, postédition et révision. S'il veut survivre en tant qu'élément moteur du processus de traduction et non comme simple rouage, le traducteur doit donc devenir lui-même un élément « critique » (dans tous les sens du terme) de l'ergonomie globale de ce processus. 


\section{BIBLIOGRAPHIE}

FULFORD Heather et GRENELL-ZAFRA Joaquin, « The Freelance Translator's Workstation: An Empirical Investigation ", dans Proceedings of the 9th European Association for Machine Translation Workshop (University of Malta, 26 avril 2004), 2004, p. 53-61. Disponible sur <http://www.mtarchive.info/EAMT-2004-Fulford.pdf> [consulté le 3 octobre 2010].

GouAdec Daniel, « Modélisation du processus d'exécution des traductions », Meta, vol. 50, $\mathrm{n}^{\circ} 2$, avril 2005, p. 643-655.

-, Profession traducteur, Paris, Maison du dictionnaire, 2009 ( $1^{\text {re }}$ édition en 2003), 432 p.

HÖCKER Mary et WHEATLEy Alan, Translation Memory Survey, 2003. Disponible sur <http:// ecolore.leeds.ac.uk/downloads/2003.05_bdue_survey_analysis.doc> [consulté le 4 octobre 2010]. KAY Martin, « The Proper Place of Men and Machines in Language Translation », Palo Alto, CA, Xerox Corporation, 1980 ; republié dans Machine Translation, vol. 12, nos 1-2, 1997, p. 3-23.

KILGRAY Translation Technologies, memoQ Quick Start Guide, 2010.

LIONBRIDGE, GeoWorkz, présentation disponible sur <https://www.geoworkz.com/Products/ FeaturesAndFunctionality.aspx - LanguageManagement> [consulté le 20 avril 2011].

ROUDAUD Brigitte, « La traduction automatique : l'ordinateur au service des traducteurs ", Meta : journal des traducteurs, vol. 37, $\mathrm{n}^{\circ} 4,1992$, p. 828-846. Disponible sur <http://id.erudit.org/ iderudit/003997ar> [consulté le 3 octobre 2010].

SDL Trados, présentation disponible sur <http://www.translationzone.com/fr/translatorproducts/sdl-trados-freelance/\#tab2> [consulté le 20 avril 2011].

SFT (Société française des traducteurs), Enquête tarifs 2009, Paris, 2010.

\section{NOTES}

1. Libre et gratuit jusqu'en 2002, devenu payant depuis.

2. Commentaire anonyme cité dans «Wordfast, un bref aperçu», disponible sur <http:// www.muraille.info/?q=content/wordfast-un-bref-aperçu> [consulté le 24 mai 2011].

\section{RÉSUMÉS}

Depuis l'article fondateur de Martin Kay (1980), décrivant la relation idéale entre le traducteur et la machine, l'ergonomie du poste de travail du traducteur s'est radicalement transformée, notamment sous l'effet de la révolution numérique. Dans le même temps, cette révolution a profondément modifié les rapports entre le traducteur, l'entreprise de traduction et le donneur 
d'ouvrage. Cet article cherche tout d'abord à définir le poste de travail idéal dans la perspective de chacun des acteurs de la chaîne de traduction. Il décrit ensuite l'évolution du poste de travail au cours des vingt dernières années et l'impact, réel ou perçu, de cette évolution sur les acteurs. Il examine enfin à travers un exemple, le rôle crucial que joue l'ergonomie des interfaces homme-machine dans la relation qu'entretient le traducteur avec sa traduction.

In his seminal article on "The Proper Place of Men and Machines in Language Translation" (1980), Martin Kay described the ideal relation between the translator and the machine. Since then, radical changes have affected and are still affecting the translator's work station, principally as a result of the digital revolution. This same revolution has profoundly changed the relationship between the translator, the translation service provider and the endclient. This article first of all attempts to define the "ideal" work station as seen from the perspective of each of the players in the translation process. It then describes the work station changes of the past twenty years and their real or perceived impact on the various players. Finally, on the basis of an example, it examines the crucial role played by man-machine interfaces in the relation between the translator and her/his translation.

INDEX

Mots-clés : ergonomie, interface homme-machine, poste de travail, traduction

Keywords : ergonomics, man-machine interface, translation, work station

\section{AUTEURS}

DANIEL TOUDIC

CFTTR, Université Rennes 2, LIDILE EA 3874

GUILLAUME DE BRÉBISSON

CFTTR, Université Rennes 2, LIDILE EA 3874 